\title{
Synthesis, Characterization and Antitumour Activity of Some Novel Oxazine Substituted 9-Anilinoacridines and their 3D-QSAR Studies
}

\author{
R. KALIRAJAN*, V. KULSHRESTHAAND S. SANKAR \\ Department of Pharmaceutical Chemistry, JSS College of Pharmacy, Udhagamandalam-643 001, India
}

Kalirajan et al:: Oxazine bearing 9-anilioacridines and Antitumour activity

\begin{abstract}
A series of oxazine substituted 9-anilinoacridines were synthesized, characterized, and evaluated for antitumor activity against Daltons lymphoma ascites cells using in vitro and in vivo methods. Results indicated that these conjugates exhibited significant antitumour activity on Daltons lymphoma ascites cells. Among these agents, compounds $4 \mathrm{~b}, 4 \mathrm{c}, 4 \mathrm{e}$ and $4 \mathrm{j}$ were the most cytotoxic with $\mathrm{CTC}_{50}$ value of $96.5-190 \mu \mathrm{g} / \mathrm{ml}$ $(0.125-0.352 \mu M)$. 3D QSAR study was performed using PHASE module of Schrodinger suite.
\end{abstract}

Key words: Acridine, Oxazine, Synthesis, Antitumour, 3D-QSAR

Chemotherapy is often the treatment of choice for many types of cancer and the search for new chemotherapeutic agents still plays a major role in the fight against cancer. A reasonable approach in this area deals with use of compounds interacting with DNA and/or inhibiting enzymes critical for cell survival and replication. Amsacrine is one such compound, a well-known antiproliferative agent used to treat some types of cancers including acute adult leukaemia ${ }^{[1]}$. The poisoning of topo II activity inhibits the relegation process and causes lethal double-strand breaks in DNA, leading to cell cycle arrest and apoptosis. The intercalative property was referred to the planar aromatic system of the acridine moiety ${ }^{[2]}$.

In the same context, acridines have gained strong ground for various biological activities like antimicrobial ${ }^{[3]}$, antioxidant ${ }^{[4]}$, anticancer ${ }^{[5-8]}$ antimalarial ${ }^{[9]}$, antiinflammatory $^{[10]}$, analgesic ${ }^{[11]}$, antileishmanial ${ }^{[12]}$, antinociceptive ${ }^{[13]}$, acetylcholinesterase inhibitory ${ }^{[14]}$ and antiherpes ${ }^{[15]}$. Amsacrine is the best known compound of 9-anilinoacridines series. It was one of the first DNA-intercalating agents to be considered as a topoisomerase II inhibitor. The intercalation process is the strongest type of reversible binding to the double helical DNA in compounds with sufficiently large coplanar aromatic chromophore. Several detailed SAR studies of acridine-based DNA-intercalating agents suggest that the mode of binding is important and the chromophore intercalate with the DNA base pairs. The chemical modification of acridines such as the introduction of different substitutions or hetero cyclic rings were allowed expansion of research on the structure activity relationship to afford new insight into molecular interactions at the receptor level ${ }^{[16]}$. In fact, it is well-established that slight structural modification on 9-anilinoacridines may bring various pharmacological effects. Similarly oxazine derivatives also have various biological activities ${ }^{[17-20]}$ like antimicrobial, anticancer. In this paper in vitro and in vivo antitumour activity against Daltons lymphoma ascites (DLA) cell lines were described. In continuation of our previous research work ${ }^{[21]}$ on searching new potent cytotoxic agents, 9-anilinoacridine analogues bearing the oxazine residue on anilino rings were synthesized for antitumour evaluation. Acridine derivatives possessed a diverse range of pharmacological activities ${ }^{[22]}$. Hence the main objective of this study was to determine the antibacterial and antitumour activities of oxazine substituted 9-anilino acridine derivatives. The results revealed that the newly synthesized derivatives exhibited significant antitumour activities.

This is an open access article distributed under the terms of the Creative Commons Attribution-NonCommercial-ShareAlike 3.0 License, which allows others to remix, tweak, and build upon the work non-commercially, as long as the author is credited and the new creations are licensed under the identical terms

Accepted 28 August 2018

Revised 26 February 2018

Received 12 April 2017

Indian J Pharm Sci 2018;80(5):921-929 


\section{MATERIALS AND METHODS}

Melting points were obtained on Veego VMP-1 apparatus in open capillary tubes and are uncorrected. The reactions were monitored by thin-layer chromatography (TLC) on silica gel thin-layer plates. Compounds were analysed for $\mathrm{C}, \mathrm{H}, \mathrm{N}$ and analytical results obtained for these elements were within $\pm 0.5 \%$ of the calculated values for the formula shown. All reagents were of commercially quality or were purified before use. Organic solvents were of analytical grade or were purified by standard procedures. IR spectra were obtained using a Perkin Elmer FT-IR spectrometer spectrum two model. ${ }^{1} \mathrm{H}$ nuclear magnetic resonance (NMR) and ${ }^{13} \mathrm{C}$ NMR were recorded on Bruker Avance III $500 \mathrm{MHz}$ spectrometer. Chemical shifts are in parts per million (ppm). Mass spectra of the final compounds were recorded on a Jeol GC-Mate mass spectrometer.

9-chloroacridine was synthesized by the cyclization of $\mathrm{N}$-arylanthranilinic acid with phosporusoxy chloride as reported ${ }^{[23]}$. 1-[4-(Acridin-9-ylamino) phenyl]ethanone (2) was synthesized by the reaction of 4-aminoacetophenone was refluxed with 9-chloroacridine as reported ${ }^{[24]}$.

\section{General procedure for synthesis of chalcones (3a-k):}

The chalcones were synthesized by using the general Claisen-Schmidt condensation ${ }^{[25]}$. In a $100 \mathrm{ml}$ flat bottomed flask, $25 \mathrm{ml}$ of the $10 \%$ sodium hydroxide and $25 \mathrm{ml}$ of ethanol were taken along with a magnetic stirring bar and was stirred on the magnetic stirrer. To this $0.01 \mathrm{~mol}$ of corresponding aldehyde was added, then $2.99 \mathrm{~g}$ (0.0096 mol) of 1-[4-(acridin-9-ylamino) phenyl]ethanone was added at the last. The solution was allowed to stir for $8 \mathrm{~h}$ at room temperature. After completion of the reaction, $100 \mathrm{ml}$ of water was added, the precipitate formed was filtered, washed three times with $50 \mathrm{ml}$ of water each time to remove sodium hydroxide, dried and crystallized from ethanol.

\section{(E)-1-(4-(acridin-9-ylamino)phenyl)-3-(4-hydroxy phenyl)prop-2-en-1-one (3a):}

Yellow powder, \% yield: 59; melting point (MP): 195198 ${ }^{\circ}$, IR (v, $\left.\mathrm{cm}^{-1}\right): 3302(\mathrm{~N}-\mathrm{H}), 3100-3000(\mathrm{Ar} \mathrm{C}-\mathrm{H})$, $1624(\alpha, \beta$-unsaturated $\mathrm{C}=\mathrm{O}), 1606$ and $1518(\mathrm{ArC}=\mathrm{C})$, 1267 (C-N), 748 (ArC-H); MS: m/z $434.52\left(\mathrm{M}^{+}\right) ;{ }^{1} \mathrm{H}$ NMR (DMSO-d6, $\delta$ ppm): 6.65-8.02 (16H, m, ArCH), 7.90 and $7.56(2 \mathrm{H}$, ss, $\mathrm{CH}=\mathrm{CH}), 11.21(1 \mathrm{H} \mathrm{s}, \mathrm{NH})$; ${ }^{13} \mathrm{C}$ NMR (DMSO-d6, $\delta$ ppm): $189(\mathrm{C}=\mathrm{O}), 145.3,120.4$ $(\mathrm{CH}=\mathrm{CH}), 153.5,150.8,148.4,148.5,143.2,141.2$, 136.3, 136.1, 132.4, 131.7, 130.8, 129.6, 130.5, 128.6,
127.2, 127.1, 127.2, 121.6, 119.5, 116.3. Anal. calcd. for $\mathrm{C}_{28} \mathrm{H}_{19} \mathrm{ClN}_{2} \mathrm{O}: \mathrm{C}, 77.32 ; \mathrm{H}, 4.42 ; \mathrm{N}, 6.45$; found: $\mathrm{C}$, $77.25 ; \mathrm{H}, 4.29 ; \mathrm{N}, 6.62$.

(E)-1-(4-(acridin-9-ylamino)phenyl)-3-(4-methoxy phenyl)prop-2-en-1-one (3b):

Yellow powder, \% yield: 58; MP: $179-181^{\circ}$, IR $\left(v, \mathrm{~cm}^{-1}\right): 3273(\mathrm{NH}), 3100-3000(\mathrm{ArCH}), 1626$ $(\alpha, \beta$-unsaturated $\mathrm{C}=\mathrm{O}), 1607$ and $1510(\mathrm{ArC}=\mathrm{C})$, $1260(\mathrm{CN}), 1168$ (CO), 748 (Ar CH); MS: m/z 430.17 (100 \%); ${ }^{1} \mathrm{H}$ NMR (DMSO-d6) $\delta: 6.65-8.02(16 \mathrm{H}, \mathrm{m}$, $\mathrm{ArCH}), 7.90$ and $7.56(2 \mathrm{H}, \mathrm{s}, \mathrm{CH}=\mathrm{CH}), 11.21(1 \mathrm{H}, \mathrm{s}$, $\mathrm{NH}), 3.73\left(3 \mathrm{H}, \mathrm{t}, \mathrm{CH}_{3}\right) ;{ }^{13} \mathrm{C} \mathrm{NMR}(\mathrm{ppm}): 189(\mathrm{C}=\mathrm{O})$, 148.8, 143.8, 141.4, 135.6, 135.5, 131.6, 131.5, 130.3, $129.6,129.8,128.9,127.7,126.8,126.7,121.9$, 119.4, 115.7, $54.8\left(\mathrm{CH}_{3}\right)$. Anal. calcd. for $\mathrm{C}_{29} \mathrm{H}_{22} \mathrm{~N}_{2} \mathrm{O}_{2}$ : C, 80.91; H, 5.15; N, 6.51; O, 7.43; found: C, 80.83; H, $5.23 ; \mathrm{N}, 6.44$.

(E)-1-(4-(acridin-9-ylamino)phenyl)-3-(3,4-di methoxyphenyl)prop-2-en-1-one (3c):

Yellow powder, \% yield: 54; MP: $228-230^{\circ}$, IR $\left(v, \mathrm{~cm}^{-1}\right): 3044(\mathrm{NH}), 3100-3000($ Ar st $\mathrm{CH}), 1626$ $(\alpha, \beta$-unsaturated $\mathrm{C}=\mathrm{O}), 1577$ and $1498(\mathrm{ArC}=\mathrm{C}), 748$ $(\mathrm{ArCH}) ; \mathrm{MS}: \mathrm{m} / \mathrm{z} 460.58\left(\mathrm{M}^{+}\right) ;{ }^{1} \mathrm{H}$ NMR (DMSO-d6, $\delta \mathrm{ppm}): 6.65-8.02(16 \mathrm{H}, \mathrm{m}, \mathrm{ArCH}), 7.90$ and 7.56 $(2 \mathrm{H}, \mathrm{s}, \mathrm{CH}=\mathrm{CH}), 11.21(1 \mathrm{H}, \mathrm{s}, \mathrm{NH}) .{ }^{13} \mathrm{C} \mathrm{NMR}(\mathrm{ppm})$ : $181(\mathrm{C}=\mathrm{O}), 53.47\left(\mathrm{OCH}_{3}\right), 54.32\left(\mathrm{OCH}_{3}\right), 104.8-158.8$ (aromatic carbons). Anal. calcd. for $\mathrm{C}_{30} \mathrm{H}_{24} \mathrm{~N}_{2} \mathrm{O}_{3}: \mathrm{C}$, 78.29; H, 5.33; N, 6.13; found: $\mathrm{C}, 78.25 ; \mathrm{H}, 5.36 ; \mathrm{N}$, 6.12 .

(E)-1-(4-(acridin-9-ylamino)phenyl)-3-(4-hydroxy3-methoxyphenyl)prop-2-en-1-one (3d):

Yellow powder, \% yield: 73; MP: 210-212 ${ }^{\circ}$, IR $\left(v, \mathrm{~cm}^{-1}\right): 3304(\mathrm{NH}), 3100-3000$ (Ar st $\left.\mathrm{CH}\right), 1626$ $(\alpha, \beta$-unsaturated $\mathrm{C}=\mathrm{O}), 1587$ and $1568(\mathrm{ArC}=\mathrm{C})$, 3327 (ArOH), 748 (Ar CH); MS: m/z $450.34\left(\mathrm{M}^{+}\right)$; ${ }^{1} \mathrm{H}$ NMR (DMSO-d6, $\delta$ ppm): 6.65-8.02 (16H, m, ArH), 7.90 and $7.56(2 \mathrm{H}, \mathrm{s}, \mathrm{CH}=\mathrm{CH}), 11.21(1 \mathrm{H}, \mathrm{s}, \mathrm{NH})$; ${ }^{13} \mathrm{C}$ NMR (ppm): $178(\mathrm{C}=\mathrm{O}), 52.15\left(\mathrm{OCH}_{3}\right), 110.2-$ 169.3 (aromatic carbons). Anal. calcd. for $\mathrm{C}_{29} \mathrm{H}_{26} \mathrm{~N}_{2} \mathrm{O}_{3}$ : C, 77.42; H, 5.85; N, 6.33; found: C, 77.46; H, 5.92; $\mathrm{N}, 6.37$.

(E)-1-(4-(acridin-9-ylamino)phenyl)-3-(furan-2-yl) prop-2-en-1-one (3e):

Yellow powder, \% yield: 67; MP: $179-181^{\circ}$, IR $\left(v, \mathrm{~cm}^{-1}\right): 3300(\mathrm{NH}), 3057-3034(\mathrm{Ar} \mathrm{CH}), 1651$ $(\alpha, \beta$-unsaturated $\mathrm{C}=\mathrm{O}), 1606$ and $1512(\mathrm{Ar} \mathrm{C}=\mathrm{C})$, $1230(\mathrm{CN}), 1176(\mathrm{CO}), 759(\mathrm{Ar} \mathrm{CH}) ; \mathrm{MS}(\mathrm{m} / \mathrm{z})$ : 
$391.14\left(\mathrm{M}^{+}+1\right)$; ${ }^{1} \mathrm{H}$ NMR (DMSO-d6, $\delta$ ppm): 8.04 to $6.64(15 \mathrm{H}, \mathrm{m}, \mathrm{ArCH}), 10.74(1 \mathrm{H}, \mathrm{s}, \mathrm{NH}), 7.58$ and 7.91 $(2 \mathrm{H}, \mathrm{ss}, \mathrm{CH}=\mathrm{CH}) ;{ }^{13} \mathrm{C}$ NMR (ppm): $180(\mathrm{C}=\mathrm{O}), 53.17$ $\left(\mathrm{OCH}_{3}\right), 105.7-167.5$ (aromatic carbons). Anal. calcd. for $\mathrm{C}_{26} \mathrm{H}_{18} \mathrm{~N}_{2} \mathrm{O}_{2}: \mathrm{C}, 79.97 ; \mathrm{H}, 4.64 ; \mathrm{N}, 7.17$; found: $\mathrm{C}$, 79.88; H, 4.53; N, 7.25.

(E)-1-(4-(acridin-9-ylamino)phenyl)-3-(pyridin-2yl)prop-2-en-1-one (3f):

Yellow powder, \% yield: 48; MP: $174-177^{\circ}$; IR $\left(v, \mathrm{~cm}^{-1}\right): 3028(\mathrm{NH}), 3100-3000 \quad(\mathrm{ArCH}), 1622$ $(\alpha, \beta$-unsaturated $\mathrm{C}=\mathrm{O}), 1579$ and $1498(\mathrm{ArC}=\mathrm{C}), 1280$ (CN), $744(\mathrm{ArCH}) ; \mathrm{MS}: \mathrm{m} / \mathrm{z} 401.47\left(\mathrm{M}^{+}\right) ;{ }^{1} \mathrm{H} \mathrm{NMR}$ (DMSO-d6, $\delta$ ppm): 6.65-8.02 (16H, m, ArCH), 7.90 and $7.56(2 \mathrm{H}, \mathrm{s}, \mathrm{CH}=\mathrm{CH}), 11.21(1 \mathrm{H}, \mathrm{s}, \mathrm{NH}) ;{ }^{13} \mathrm{C} \mathrm{NMR}$ (ppm): $183(\mathrm{C}=\mathrm{O}), 112.5-153.6$ (aromatic carbons). Anal. calcd. for $\mathrm{C}_{27} \mathrm{H}_{19} \mathrm{~N}_{3} \mathrm{O}: \mathrm{C}, 76.47 ; \mathrm{H}, 4.30 ; \mathrm{N}, 9.45$; found: C, 76.26; H, 4.52; N, 9.51.

(E)-1-(4-(acridin-9-ylamino)phenyl)-3-(pyridin-3yl)prop-2-en-1-one (3g):

Yellow powder, \% yield: 63; MP: $124-126^{\circ}$, IR $\left(v, \mathrm{~cm}^{-1}\right): 3290(\mathrm{NH}), 3100-3000 \quad(\mathrm{ArCH}), 1627$ $(\alpha, \beta$-unsaturated $\mathrm{C}=\mathrm{O}), 1577$ and $1498(\mathrm{ArC}=\mathrm{C})$, 1280 (CN), 748 (ArCH); MS (m/z): $402.15\left(\mathrm{M}^{+}+1\right) ;{ }^{1} \mathrm{H}$ NMR (DMSO-d6, $\delta$ ppm): 3.98 (s, 1H, NH), 7.58 (s, $1 \mathrm{H}, \mathrm{CH}), 7.89(\mathrm{~s}, 1 \mathrm{H}, \mathrm{CH}), 8.1$ to $6.63(15 \mathrm{H}, \mathrm{m}, \mathrm{ArCH})$. ${ }^{13} \mathrm{C}$ NMR (ppm): $181(\mathrm{C}=\mathrm{O}), 110.3-157.3$ (aromatic carbons). Anal. calcd. for $\mathrm{C}_{27} \mathrm{H}_{19} \mathrm{~N}_{3} \mathrm{O}$ (401.4): C, 80.80; H, 4.77; N, 10.48; found: C, 80.67; H, 4.60; N, 10.28.

(E)-1-(4-(acridin-9-ylamino)phenyl)-3-(pyridin-4yl)prop-2-en-1-one (3h):

Yellow powder, $\%$ yield: 68 ; MP: $165-170^{\circ}$, IR (v, $\left.\mathrm{cm}^{-1}\right): 3308(\mathrm{NH}), 3100-3000(\mathrm{Ar}$ st $\mathrm{CH}), 1626$ $(\alpha, \beta$-unsaturated $\mathrm{C}=\mathrm{O}), 1587$ and $1568(\mathrm{ArC}=\mathrm{C}), 748$ (ArCH); MS: m/z $401.48\left(\mathrm{M}^{+}\right)$; ${ }^{1} \mathrm{H}$ NMR (DMSO-d6, $\delta \mathrm{ppm}): 6.60-8.08(16 \mathrm{H}, \mathrm{m}, \mathrm{ArCH}), 7.90$ and 7.56 $(2 \mathrm{H}, \mathrm{s}, \mathrm{CH}=\mathrm{CH}), 11.21(1 \mathrm{H}, \mathrm{s}, \mathrm{NH}) ;{ }^{13} \mathrm{C} \mathrm{NMR}(\mathrm{ppm})$ : $179(\mathrm{C}=\mathrm{O}), 110.4-153.7$ (aromatic carbons). Anal. calcd. for $\mathrm{C}_{27} \mathrm{H}_{19} \mathrm{~N}_{3} \mathrm{O}: \mathrm{C}, 78.42 ; \mathrm{H}, 5.85 ; \mathrm{N}, 6.33$; found: C, 78.46; H, 5.92; N, 6.37.

(E)-1-(4-(acridin-9-ylamino)phenyl)but-2-en-1-one (3i):

Yellow powder, \% yield: 61; MP: $188-190^{\circ}$, IR (v, $\left.\mathrm{cm}^{-1}\right): 3347(\mathrm{NH}), 3100-3000($ Ar st $\mathrm{CH}), 1622$ $(\alpha, \beta$-unsaturated $\mathrm{C}=\mathrm{O}), 1604$ and $1473(\mathrm{ArC}=\mathrm{C})$; MS: m/z 338.18 $\left(\mathrm{M}^{+}\right)$; ${ }^{1} \mathrm{H}$ NMR (DMSO-d6, $\delta \mathrm{ppm}$ ): $3.72\left(\mathrm{CH}_{3}\right), 6.65-8.02(16 \mathrm{H}, \mathrm{m}, \mathrm{ArCH}), 7.90$ and 7.56 $(2 \mathrm{H}, \mathrm{s}, \mathrm{CH}=\mathrm{CH}), 11.21(1 \mathrm{H}, \mathrm{s}, \mathrm{NH}) ;{ }^{13} \mathrm{C}$ NMR $(\mathrm{ppm})$ :
$185(\mathrm{C}=\mathrm{O}), \quad 54.32\left(\mathrm{CH}_{3}\right), \quad 114.8-154.5 \quad$ (aromatic carbons). Anal. calcd. for $\mathrm{C}_{23} \mathrm{H}_{18} \mathrm{~N}_{2} \mathrm{O}: \mathrm{C}, 81.49 ; \mathrm{H}$, 5.33; N, 8.23; found: $\mathrm{C}, 81.53 ; \mathrm{H}, 5.37 ; \mathrm{N}, 8.13$.

(E)-1-(4-(acridin-9-ylamino)phenyl)pent-2-en-1one $(3 \mathbf{j})$ :

Yellow powder, \% yield: 54; MP: 195-200 IR (v, $\left.\mathrm{cm}^{-1}\right): 3044(\mathrm{NH}), 3100-3000($ Ar st CH), 1626 $(\alpha, \beta$-unsaturated $\mathrm{C}=\mathrm{O}), 1582$ and $1496(\mathrm{ArC}=\mathrm{C}), 754$ (ArCH); MS: m/z $352.43\left(\mathrm{M}^{+}\right)$; ${ }^{1} \mathrm{H}$ NMR (DMSO-d6, $\delta$ ppm): $3.72\left(\mathrm{CH}_{3}\right), 3.32\left(\mathrm{CH}_{3}\right), 3.62\left(\mathrm{CH}_{2}\right), 6.65-8.02$ $(16 \mathrm{H}, \mathrm{m}, \mathrm{ArCH}), 7.90$ and $7.56(2 \mathrm{H}, \mathrm{s}, \mathrm{CH}=\mathrm{CH}), 11.21$ (1H, s, NH). ${ }^{13} \mathrm{C}$ NMR (ppm): $181(\mathrm{C}=\mathrm{O}), 104.8-158.6$ (aromatic carbons). Anal. calcd. for $\mathrm{C}_{24} \mathrm{H}_{20} \mathrm{~N}_{2} \mathrm{O}: \mathrm{C}$, 74.29; H, 5.33; N, 6.13; found: C, 74.25; H, 5.36; N, 6.12 .

(2E,4E)-1-(4-(acridin-9-ylamino)phenyl)hexa-2,4dien-1-one (3k):

Yellow powder, \% yield: 76 ; MP: $178-184^{\circ}$, IR $\left(v, \mathrm{~cm}^{-1}\right): 3058(\mathrm{NH}), 3100-3000($ Ar st $\mathrm{CH}), 1632$ $(\alpha, \beta$-unsaturated $\mathrm{C}=\mathrm{O}), 1595$ and $1478(\mathrm{ArC}=\mathrm{C}), 758$ (ArCH); MS: m/z $364.48\left(\mathrm{M}^{+}\right)$; ${ }^{1} \mathrm{H}$ NMR (DMSO-d6, $\delta$ ppm): $3.32\left(\mathrm{CH}_{3}\right), 6.65-8.02(16 \mathrm{H}, \mathrm{m}, \mathrm{ArCH}), 7.90$ and $7.56(2 \mathrm{H}, \mathrm{CH}=\mathrm{CH}), 11.21(1 \mathrm{H}, \mathrm{s}, \mathrm{NH}) .{ }^{13} \mathrm{C} \mathrm{NMR}$ (ppm): $183(\mathrm{C}=\mathrm{O}), 113.5-155.9$ (aromatic carbons). Anal. calcd. for $\mathrm{C}_{25} \mathrm{H}_{20} \mathrm{~N}_{2} \mathrm{O}: \mathrm{C}, 76.29 ; \mathrm{H}, 5.33 ; \mathrm{N}, 6.13$; found: $\mathrm{C}, 76.25 ; \mathrm{H}, 5.36 ; \mathrm{N}, 6.23$.

General procedure for synthesis of oxazine substituted 9-anilinoacridines (4a-k):

A mixture of chalcone $3 \mathrm{a}-\mathrm{k}(0.02 \mathrm{~mol})$, urea $(0.02 \mathrm{~mol})$ were dissolved in sodium hydroxide in ethanol $(10 \mathrm{ml})$, stirred for about for 2-3 h on a magnetic stirrer. This mixture was poured into $400 \mathrm{ml}$ of cold water with continuous stirring for $1 \mathrm{~h}$. This was kept in refrigerator for $24 \mathrm{~h}$. The precipitate obtained was filtered, washed and recrystallized using petroleum ether:benzene (5:5). The reaction was monitored by TLC using methanol:water (5:3).

\section{4-(6-(4-(acridin-9-ylamino)phenyl)-2-amino-2H- 1,3-oxazin-4-yl)phenol (4a):}

Yellow powder, \% yield: 54; MP: $108-111^{\circ}$, IR (v, $\left.\mathrm{cm}^{-1}\right): 3389\left(\mathrm{NH}_{2}\right), 3328(\mathrm{NH}), 1586$ and $1437(\mathrm{ArC}=\mathrm{C})$, $1176(\mathrm{ArC}=\mathrm{N}), 3223(\mathrm{Ar}-\mathrm{OH}), 819(\mathrm{ArCH}) ; \mathrm{MS}: \mathrm{m} / \mathrm{z}$ 458. $51\left(\mathrm{M}^{+}\right) ;{ }^{1} \mathrm{H}$ NMR (DMSO-d6, $\delta \mathrm{ppm}$ ): 5.62 (s, 1H, OH), 6.85-7.94 (16H, m, ArCH), 7.46-7.48 $(2 \mathrm{H}, \mathrm{d}, \mathrm{CH}), 7.95(1 \mathrm{H}, \mathrm{s}, \mathrm{NH}), 6.12\left(2 \mathrm{H}, \mathrm{s}, \mathrm{NH}_{2}\right)$; ${ }^{13} \mathrm{C}$ NMR (ppm): 179.7 169.6, 163.7, 150.2, 148.3, 148.5, 145.3, 120.5, 142.5, 141.2, 136.2, 136.1, 132.5, 
$131.5,130.8,130.5,129.6,128.8,127.3,127.2$, 127.1, $121.6,119.5,116.3,116.2,114.65,112.43$ (aromatic carbons). Anal. calc. for $\mathrm{C}_{29} \mathrm{H}_{22} \mathrm{~N}_{4} \mathrm{O}_{2}: \mathrm{C}, 75.24 ; \mathrm{H}, 4.83$; N, 12.26; found: C, 75.18; H, 4.79; N, 12.31 .

N-(4-(2-amino-4-(4-methoxyphenyl)-2 H-1,3oxazin-6-yl)phenyl)acridin-9-amine (4b):

Yellow powder, \% yield: 72 ; MP: $142-145^{\circ}, \operatorname{IR}\left(v, \mathrm{~cm}^{-1}\right)$ : $3014(\mathrm{NH}), 2961$ (Ar st CH), 1588 and $1468(\mathrm{ArC}=\mathrm{C})$, $1177(\mathrm{ArC}=\mathrm{N}), 1295(\mathrm{C}-\mathrm{O}), 820(\mathrm{ArCH}) ; \mathrm{MS}: \mathrm{m} / \mathrm{z}$ $472.54\left(\mathrm{M}^{+}\right)$; ${ }^{1} \mathrm{H}$ NMR (DMSO-d6, $\left.\delta \mathrm{ppm}\right): 3.36,(3 \mathrm{H}$, $\left.\mathrm{OCH}_{3}\right), 6.12-7.39(16 \mathrm{H}, \mathrm{m}, \mathrm{ArCH}), 7.46-7.48(2 \mathrm{H}, \mathrm{m}$, $\mathrm{CH}), 11.25(1 \mathrm{H}, \mathrm{s}, \mathrm{NH}), 6.12\left(2 \mathrm{H}, \mathrm{s}, \mathrm{NH}_{2}\right) ;{ }^{13} \mathrm{C} \mathrm{NMR}$ (ppm): 166.1, 164.7, 150.4, 149.3, 148.4, 145.1, 142.5, $140.2,137.3,136.5,133.5,132.1,130.8,130.2,129.6$, $128.5,127.3,127.2,126.8,121.2,120.5,119.1,116.3$, 116.1, 114.6, 112.4 (aromatic carbons), $54.63\left(\mathrm{OCH}_{3}\right)$. Anal. calc. for $\mathrm{C}_{30} \mathrm{H}_{24} \mathrm{~N}_{4} \mathrm{O}_{2}: \mathrm{C}, 76.27 ; \mathrm{H}, 5.14 ; \mathrm{N}, 11.87$; found: $\mathrm{C}, 76.32 ; \mathrm{H}, 5.17 ; \mathrm{N}, 11.91$.

N-(4-(2-amino-4-(3,4-dimethoxyphenyl)-2H-1,3oxazin-6yl)phenyl)acridin-9-amine (4c):

Yellow powder, \% yield: 67; MP: $162-165^{\circ}$, IR (v, $\left.\mathrm{cm}^{-1}\right): 3352(\mathrm{NH}), 3005$ (Ar st CH), 1600 and 1583 $(\mathrm{ArC}=\mathrm{C}), 1642(\mathrm{ArC}=\mathrm{N}), 1263(\mathrm{CO}), 744(\mathrm{ArCH})$; MS: m/z 502.52 $\left(\mathrm{M}^{+}\right) ;{ }^{1} \mathrm{H}$ NMR (DMSO-d6, $\left.\delta \mathrm{ppm}\right)$ : 3.80, $3.86\left(6 \mathrm{H}, \mathrm{d}, \mathrm{OCH}_{3}\right), 6.85-7.97(16 \mathrm{H}, \mathrm{s}, \mathrm{ArCH})$, $7.48(1 \mathrm{H}, \mathrm{s}, \mathrm{NH}), 6.12\left(2 \mathrm{H}, \mathrm{s}, \mathrm{NH}_{2}\right) ;{ }^{13} \mathrm{C} \mathrm{NMR}(\mathrm{ppm})$ : $185.96,167.1,153.66,150.71,148.9,145.1,142.5$, $141.87,137.3,136.5,133.6,132.2,130.8,130.9$, $129.6,128.5,127.3,127.92,125.55,123.21,120.5$, 119.9, 112.7, 111.54, 110.49, (aromatic carbons), 55.69 $\left(\mathrm{OCH}_{3}\right), 55.53\left(\mathrm{OCH}_{3}\right)$. Anal. calc. for $\mathrm{C}_{31} \mathrm{H}_{26} \mathrm{~N}_{4} \mathrm{O}_{3}: \mathrm{C}$, 74.18; H, 5.16; N, 11.12; found: C, 74.23; H, 5.12; N, 11.18 .

4-(6-(4-(acridin-9-ylamino)phenyl)-2-amino-2H1,3-oxazin-4yl)-2methoxyphenol (4d):

Yellow powder, \% yield: 57; MP: $169-172^{\circ}$, IR $\left(v, \mathrm{~cm}^{-1}\right): 3332(\mathrm{NH}), 2993(\mathrm{Ar}$ st $\mathrm{CH}), 1589$ and $1563(\mathrm{ArC}=\mathrm{C}), 3227(\mathrm{Ar}-\mathrm{OH}), 1653(\mathrm{ArC}=\mathrm{N}), 1178$ (C-O), 750 (ArC-H); MS: m/z $488.47\left(\mathrm{M}^{+}\right) ;{ }^{1} \mathrm{H}$ NMR (DMSO-d6, $\delta$ ppm): 3.36, (3H, d, $\left.\mathrm{OCH}_{3}\right), 5.34(\mathrm{~s}, 1 \mathrm{H}$, $\mathrm{OH}), 7.27-7.71(18 \mathrm{H}, \mathrm{m}, \mathrm{ArH}), 8.28(1 \mathrm{H}, \mathrm{s}, \mathrm{NH}), 6.27$ $\left(2 \mathrm{H}, \mathrm{s}, \mathrm{NH}_{2}\right) ;{ }^{13} \mathrm{C} \mathrm{NMR}(\mathrm{ppm}): 40.03\left(\mathrm{OCH}_{3}\right), 163.9$, 161.7, 151.3, 149.4, 147.1, 145.3, 141.5, 140.3, 138.5, $136.2,133.1,132.5,130.8,130.1,129.3,128.2,127.3$, 127.1, 126.3, 121.6, 120.2, 119.2, 117.3, 116.8, 114.6, 112.4 (aromatic carbons). Anal. calc. For $\mathrm{C}_{30} \mathrm{H}_{24} \mathrm{~N}_{4} \mathrm{O}_{3}$ : C, 73.68; H, 4.87; N, 11.36; found: C, 73.68; H, 4.87; N, 11.36 .
N-(4-(2-amino-4-(furan-2-yl)-2H-1,3-oxazin-6-yl) phenyl)acridin-9-amine (4e):

Orange powder, $\%$ yield: 61 ; MP: $110-113^{\circ}$; yield: $\%$, IR (v, cm-1): $3415\left(\mathrm{NH}_{2}\right), 3348(\mathrm{NH}), 1599$ and 1477 $(\mathrm{ArC}=\mathrm{C}), 1632(\mathrm{ArC}=\mathrm{N}), 1259(\mathrm{CO}), 881(\mathrm{ArCH})$; MS: m/z $432.47\left(\mathrm{M}^{+}\right) ;{ }^{1} \mathrm{H}$ NMR (DMSO-d6, $\delta \mathrm{ppm}$ ): 6.24-7.98 (18H, m, ArH), $8.22(1 \mathrm{H}, \mathrm{s}, \mathrm{NH}), 6.24$ $\left(2 \mathrm{H}, \mathrm{s}, \mathrm{NH}_{2}\right) ;{ }^{13} \mathrm{C}$ NMR (ppm): 170.2, 168.1, 166.7, 151.7, 149.5, 148.1, 145.6, 142.2, 140.7, 138.2, 136.2, $134.5,132.7,130.5,130.1,129.3,128.3,127.7,127.2$, 126.6, 121.4, 120.4, 119.8, 116.7, 116.1, 115.6, 112.4, 108.8 (aromatic carbons). Anal. calc. for $\mathrm{C}_{27} \mathrm{H}_{20} \mathrm{~N}_{4} \mathrm{O}_{2}$ : C, 74.96; H, 4.68; N, 12.87; found: C, 74.93; H, 4.72; $\mathrm{N}, 12.83$.

N-(4-(2-amino-4-(pyridin-2-yl)-2H-1,3-oxazin-6-yl) phenyl)acridin-9-amine (4f):

Brown powder, \% yield: 55; MP: 200-203; IR (v, $\left.\mathrm{cm}^{-1}\right)$ : $3349\left(\mathrm{NH}_{2}\right), 3224(\mathrm{NH}), 1586$ and $1473(\mathrm{ArC}=\mathrm{C})$, $1636(\mathrm{ArC}=\mathrm{N}), 1259(\mathrm{CO}), 748(\mathrm{ArCH}) . \mathrm{MS}: \mathrm{m} / \mathrm{z}$ $443.47\left(\mathrm{M}^{+}\right)$; ${ }^{1} \mathrm{H}$ NMR (DMSO-d6, $\left.\delta \mathrm{ppm}\right)$ : 6.06-8.49 $(16 \mathrm{H}, \mathrm{m}, \mathrm{ArCH}), 8.40-8.43(2 \mathrm{H}, \mathrm{m}, \mathrm{CH}), 5.45(1 \mathrm{H}, \mathrm{s}$, $\mathrm{NH}), 4.54$ (2H, s, $\left.\mathrm{NH}_{2}\right) ;{ }^{13} \mathrm{C} \mathrm{NMR}$ (ppm): 168.4, 167.3, 153.7, 150.5, 147.3, 145.2, 142.1, 141.4, 138.6, 136.5, $134.3,132.7,131.3,130.1,129.5,128.8,127.4,127.2$, 126.2, 121.1, 120.7, 119.2, 116.7, 116.4, 114.2, 112.7, 109.3 (aromatic carbons). Anal. calc. for $\mathrm{C}_{28} \mathrm{H}_{21} \mathrm{~N}_{5} \mathrm{O}: \mathrm{C}$, 75.87; H, 4.74; N, 15.79; found: C, 75.87; H, 4.74; N, 15.79 .

N-(4-(2-amino-4-(pyridin-3-yl)-2H-1,3-oxazin-6-yl) phenyl)acridin-9-amine (4g):

Brown powder, $\%$ yield: 53; MP: $135-137^{\circ}$, IR (v, $\left.\mathrm{cm}^{-1}\right): 3347\left(\mathrm{NH}_{2}\right), 3221(\mathrm{NH}), 1587$ and $1478(\mathrm{ArC}=\mathrm{C})$, $1632(\mathrm{ArC}=\mathrm{N}), 1278(\mathrm{CO}), 827(\mathrm{ArCH}) ; \mathrm{MS}: \mathrm{m} / \mathrm{z}$ $443.47\left(\mathrm{M}^{+}\right)$; ${ }^{1} \mathrm{H}$ NMR (DMSO-d6, $\delta \mathrm{ppm}$ ): 6.128.47 (16H, m, ArCH), 8.38-8.42 (2H, m, CH), 5.75 $(1 \mathrm{H}, \mathrm{s}, \mathrm{NH}), 4.76\left(2 \mathrm{H}, \mathrm{s}, \mathrm{NH}_{2}\right) ;{ }^{13} \mathrm{C} \mathrm{NMR}$ (in ppm): 171.5, 167.4, 155.3, 151.3, 147.1, 145.2, 142.5, 141.5, $138.3,137.5,134.2,132.5,131.7,130.4,129.9,128.5$, 127.6, 127.3, 126.7, 121.5, 120.3, 119.6, 117.4, 116.7, 114.5, 112.2, 109.6, 107.4 (aromatic carbons). Anal. calc. for $\mathrm{C}_{28} \mathrm{H}_{21} \mathrm{~N}_{5} \mathrm{O}: \mathrm{C}, 75.81 ; \mathrm{H}, 4.75 ; \mathrm{N}, 15.78$; found: C, 75.83; H, 4.74; N, 15.75.

N-(4-(2-amino-4-(pyridin-4-yl)-2H-1,3-oxazin-6-yl) phenyl)acridin-9-amine (4h):

Brown powder, \% yield: 51; MP: $158-161^{\circ}, \operatorname{IR}\left(v, \mathrm{~cm}^{-1}\right)$ : $3346\left(\mathrm{NH}_{2}\right), 3219(\mathrm{NH}), 1587$ and $1477(\mathrm{ArC}=\mathrm{C}), 1632$ $(\mathrm{ArC}=\mathrm{N}), 1225(\mathrm{CO}), 822(\mathrm{ArCH}) ; \mathrm{MS}: \mathrm{m} / \mathrm{z} 443.47$ $\left(\mathrm{M}^{+}\right)$; ${ }^{1} \mathrm{H}$ NMR (DMSO-d6, $\delta$ ppm): 6.16-8.43 (16H, 
m, ArCH), 8.38-8.40 (2H, m, CH), $5.37(1 \mathrm{H}, \mathrm{s}, \mathrm{NH})$, $4.78\left(2 \mathrm{H}, \mathrm{s}, \mathrm{NH}_{2}\right) ;{ }^{13} \mathrm{C}$ NMR (ppm): 173.3 169.5, 167.6, 155.6, 151.8, 147.5, 144.2, 142.8, 141.1, 138.5, 136.7, 135.3, 134.6, 131.8, 130.3, 129.5, 128.5, 127.4, 126.8, $126.1,122.5,121.7,119.6,117.7,116.4,115.2,113.1$, 108.8 (aromatic carbons). Anal. calc. for $\mathrm{C}_{28} \mathrm{H}_{21} \mathrm{~N}_{5} \mathrm{O}$ : C, 75.81; H, 4.79; N, 15.78; found: C, 75.83; H, 4.76; N, 15.75 .

\section{N-(4-(2-amino-4-(methyl)-2 H-1,3-oxazin-6yl) phenyl)acridin-9-amine (4i):}

Yellow powder, \% yield: 51; MP: $173-176^{\circ}$, IR $\left(v, \mathrm{~cm}^{-1}\right): 3343(\mathrm{NH}), 3067$ (Ar st CH), 1589 and 1563 $(\mathrm{ArC}=\mathrm{C}), 1159(\mathrm{ArC}=\mathrm{N}), 1178(\mathrm{CO}), 742(\mathrm{ArCH})$; MS: m/z 380.47 $\left(\mathrm{M}^{+}\right)$; ${ }^{1} \mathrm{H}$ NMR (DMSO-d6, $\delta$ ppm): $2.50\left(3 \mathrm{H}, \mathrm{m}, \mathrm{CH}_{3}\right), 7.23-7.85(16 \mathrm{H}, \mathrm{m}, \mathrm{ArCH}), 7.59-$ $7.92(2 \mathrm{H}, \mathrm{m}, \mathrm{CH}), 8.38(1 \mathrm{H}, \mathrm{s}, \mathrm{NH}), 6.18\left(2 \mathrm{H}, \mathrm{s}, \mathrm{NH}_{2}\right)$; ${ }^{13} \mathrm{C}$ NMR (ppm): 175.2, 167.3, 153.7, 147.3, 145.2, 142.1, 141.4, 138.6, 136.5, 134.3, 132.7, 132.3, 130.5, $129.2,128.5,127.6,127.3,126.2,121.7,119.3,118.7$, 112.6, 109.5 (aromatic carbons), $54.53\left(\mathrm{CH}_{3}\right)$. Anal. calc. for $\mathrm{C}_{24} \mathrm{H}_{20} \mathrm{~N}_{4} \mathrm{O}: \mathrm{C}, 75.75 ; \mathrm{H}, 5.34 ; \mathrm{N}, 14.68$; found: C, 75.68; H, 5.41; N, 14.62 .

\section{N-(4-(2-amino-4-(ethyl)-2H-1,3-oxazin-6yl)phenyl) acridin-9-amine $(4 \mathbf{j})$ :}

Brown powder, \% yield: 49; MP: $118-121^{\circ}$, IR (v, $\left.\mathrm{cm}^{-1}\right): 3428\left(\mathrm{NH}_{2}\right), 3342(\mathrm{NH}), 1590$ and $1442(\mathrm{ArC}=\mathrm{C})$, $1626(\mathrm{ArC}=\mathrm{N}), 1279(\mathrm{CO}), 829(\mathrm{ArCH}) ; \mathrm{MS}: \mathrm{m} / \mathrm{z}$ $394.47\left(\mathrm{M}^{+}\right)$; ${ }^{1} \mathrm{H}$ NMR (DMSO-d6, $\left.\delta \mathrm{ppm}\right): 2.55$, (3H, $\left.\mathrm{m}, \mathrm{CH}_{3}\right), 2.86,\left(2 \mathrm{H}, \mathrm{s}, \mathrm{CH}_{2}\right), 6.58-7.62(16 \mathrm{H}, \mathrm{m}, \mathrm{ArH})$, 7.67-7.85 (2H, m, CH), $7.86(1 \mathrm{H}, \mathrm{s}, \mathrm{NH}), 6.18(2 \mathrm{H}$, $\left.\mathrm{s}, \mathrm{NH}_{2}\right) ;{ }^{13} \mathrm{C}$ NMR (ppm): 175.2, 168.5, 157.2, 151.3, 148.2, 143.2, 142.3, 141.3, 138.7, 136.4, 135.5, 134.2, $131.3,130.7,129.3,128.2,127.2,126.5,124.5,121.3$, 119.6, 115.2, 112.8 (aromatic carbons), $57.6\left(\mathrm{CH}_{2}\right)$, $55.32\left(\mathrm{CH}_{3}\right)$. Anal. calc. for $\mathrm{C}_{25} \mathrm{H}_{22} \mathrm{~N}_{4} \mathrm{O}: \mathrm{C}, 76.15$; H, 5.64; N, 14.18; found: C, 76.18; H, 5.67; N, 14.15.

N-(4-(2-amino-4-(prop-1-enyl)-2H-1,3-oxazin-6yl) phenyl)acridin-9-amine (4k):

Brown powder, \% yield: 53; MP: $165-167^{\circ}$; IR (v, $\left.\mathrm{cm}^{-1}\right): 3345(\mathrm{NH}), 1591$ and $1479(\mathrm{ArC}=\mathrm{C}), 1649$ $(\mathrm{ArC}=\mathrm{N}), 1278(\mathrm{CO}), 823(\mathrm{ArCH}) ; \mathrm{MS}: \mathrm{m} / \mathrm{z} 406.47$ $\left(\mathrm{M}^{+}\right) ;{ }^{1} \mathrm{H}$ NMR (DMSO-d6, $\left.\delta \mathrm{ppm}\right): 3.23\left(3 \mathrm{H}, \mathrm{s}, \mathrm{CH}_{3}\right)$, $2.85(2 \mathrm{H}, \mathrm{CH}), 7.23-7.85(18 \mathrm{H}, \mathrm{m}, \mathrm{ArH}), 8.38(1 \mathrm{H}, \mathrm{s}$, $\mathrm{NH}), 6.18\left(2 \mathrm{H}, \mathrm{s}, \mathrm{NH}_{2}\right) ;{ }^{13} \mathrm{C} \mathrm{NMR}(\mathrm{ppm}): 175.4,169.5$, 157.8, 153.4, 148.6, 143.7, 142.2, 141.1, 138.5, 136.1, 135.7, 134.3, 131.6, 130.3, 129.7, 127.9, 127.2, 126.8, 123.4, 121.1, 116.5, 114.2, 112.7 (aromatic carbons), 53.6, 54.1, 50.45 (aliphatic carbons). Anal. calc. for
$\mathrm{C}_{26} \mathrm{H}_{22} \mathrm{~N}_{4} \mathrm{O}: \mathrm{C}, 76.79 ; \mathrm{H}, 5.46 ; \mathrm{N}, 13.78$; found: $\mathrm{C}$, 76.77; H, 5.48; N, 13.75.

\section{Pharmacological evaluation:}

All the oxazine substituted 9-anilinoacridine derivatives 4a-k were screened for antibacterial activity and short term in vitro antitumour activity against DLA cells. All the synthesized final compounds 4a-k exhibited significant cytotoxic activities. The compounds $4 \mathrm{~b}$ and $4 \mathrm{~h}$ were further screened for in vivo antitumour activity against DLA cells.

\section{Short-term study for in vitro antitumor activity ${ }^{[26]}$ :}

Short term in vitro antitumor activity of the compounds was assayed by determining the percent viability of DLA cells using trypan blue dye exclusion technique. DLA cells were cultured in the peritoneal cavity of healthy albino mice weighing $25-30 \mathrm{~g}$ by injecting a suspension of DLA cells $\left(1 \times 10^{6}\right.$ cells $\left./ \mathrm{ml}\right)$ intraperitoneally. The cells were aspirated aseptically from the peritoneal cavity of the mice on $\mathrm{d} 15$. The cells were washed with Hank's balanced salt solution (HBSS) and centrifuged for $10-15 \mathrm{~min}$ at $1500 \mathrm{rpm}$ in the cooling centrifuge. The pellet was re-suspended with HBSS and the process was repeated three times. Finally the cells were suspended in a known quantity of HBSS and the cell count was adjusted to $2 \times 10^{6}$ cells $/ \mathrm{ml}$. $0.1 \mathrm{ml}$ of the diluted cell suspension was distributed in to Eppendorf tubes and exposed $0.1 \mathrm{ml}$ each of the different concentration of the drug in phosphate buffer saline and incubated at $37^{\circ}, 5 \% \mathrm{CO}_{2}$ for $3 \mathrm{~h}$. After $3 \mathrm{~h}$, trypan blue dye exclusion test was performed to determine percent viability. For testing viability using dye exclusion method, the pooled cells from wells of each concentration were mixed with $0.4 \%$ trypan blue in a ratio of $1: 1$ and the number of stained, nonstained and total number of cells were counted using haemocytometer. The percent inhibition and $\mathrm{CTC}_{50}$ values were calculated.

\section{In vivo antitumor activity:}

In vivo antitumor activities of selected compounds were carried out using DLA tumor model in mice ${ }^{[26]}$. Male Swiss albino mice were divided into 7 groups of 6 animals each. Except normal group (group 1) all the animals were injected intraperitoneally (i.p.) with $1 \times 10^{-6}$ DLA cells. Group-1 and group 2 animals received vehicle $(0.5 \% \mathrm{CMC}, 10 \mathrm{ml} / \mathrm{kg}$, p.o) and served as a normal and control, respectively. Group-3 animals received 5-fluorouracil (10 mg/ 
$\mathrm{kg}$, i.p) and treated as standard group. Group 4 and 5 animals received compound $4 \mathrm{~b}$ at a dose of 10 and $20 \mathrm{mg} / \mathrm{kg}$, p.o., respectively. Group 6 and 7 animals received compound $4 \mathrm{~h}$ at a dose of 10 and $20 \mathrm{mg} /$ $\mathrm{kg}$, p.o., respectively. The treatment was started $24 \mathrm{~h}$ after tumour inoculation and continued for a period of $24 \mathrm{~d}$. Through body weight analysis in each group, mean survival time (MST) and increase in the life span was calculated. The treatment protocols received approval from the Institutional Animal Ethics Committee.

\section{RESULTS AND DISCUSSION}

The reaction sequences leading to the various oxazine substituted 9-anilinoacridines were outlined in the fig. 1. This synthetic pathway was based on the preparation of oxazine substituted 9-anilino acridines $4 \mathrm{a}-\mathrm{k}^{[25]}$ from 9-chloroacridine 1. 1-(4-(acridine-9ylamino)phenylethanone $2^{[23]}$ was prepared from compound 1, which was refluxed with p-aminoacetophenone. The various chalcone substituted 9-anilinoacridines $3 \mathrm{a}-\mathrm{k}^{[21]}$ were prepared by the reaction of 2 with various aldehydes and these chalcone derivatives were allowed to cyclized with urea afford the corresponding oxazine substituted 9-anilinoacridines 4a-k. Synthesis, characterization and evaluation of biological activities of novel oxazine substituted 9-anilino acridines are described in this paper. The synthesized compounds were purified by column chromatography. The final yield of the derivatives was in the range of $48-73 \%$. The compounds obtained were stable in the solid as well as in the solution state. The new compounds were completely characterized by IR, ${ }^{1} \mathrm{H}$ NMR, ${ }^{13} \mathrm{C}$ NMR, mass spectral data and elemental analysis. The IR spectra of compounds 4a-k showed intense bands in the region $1200-1300 \mathrm{~cm}^{-1}$ due to carbonyl stretching and broad bands in the region $3200-3400 \mathrm{~cm}^{-1}$ due to $\mathrm{NH}$ stretching. The ${ }^{1} \mathrm{H}$ NMR spectra also support the structure of the compounds $4 \mathrm{a}-\mathrm{k}$. The $\mathrm{NH}$ proton appeared at 7.9-8.1 and $\mathrm{NH}_{2}$ proton at 5.9-6.3. The mass spectra of all compounds $4 \mathrm{a}-\mathrm{k}$ showed molecular ion peaks confirming their molecular weight.
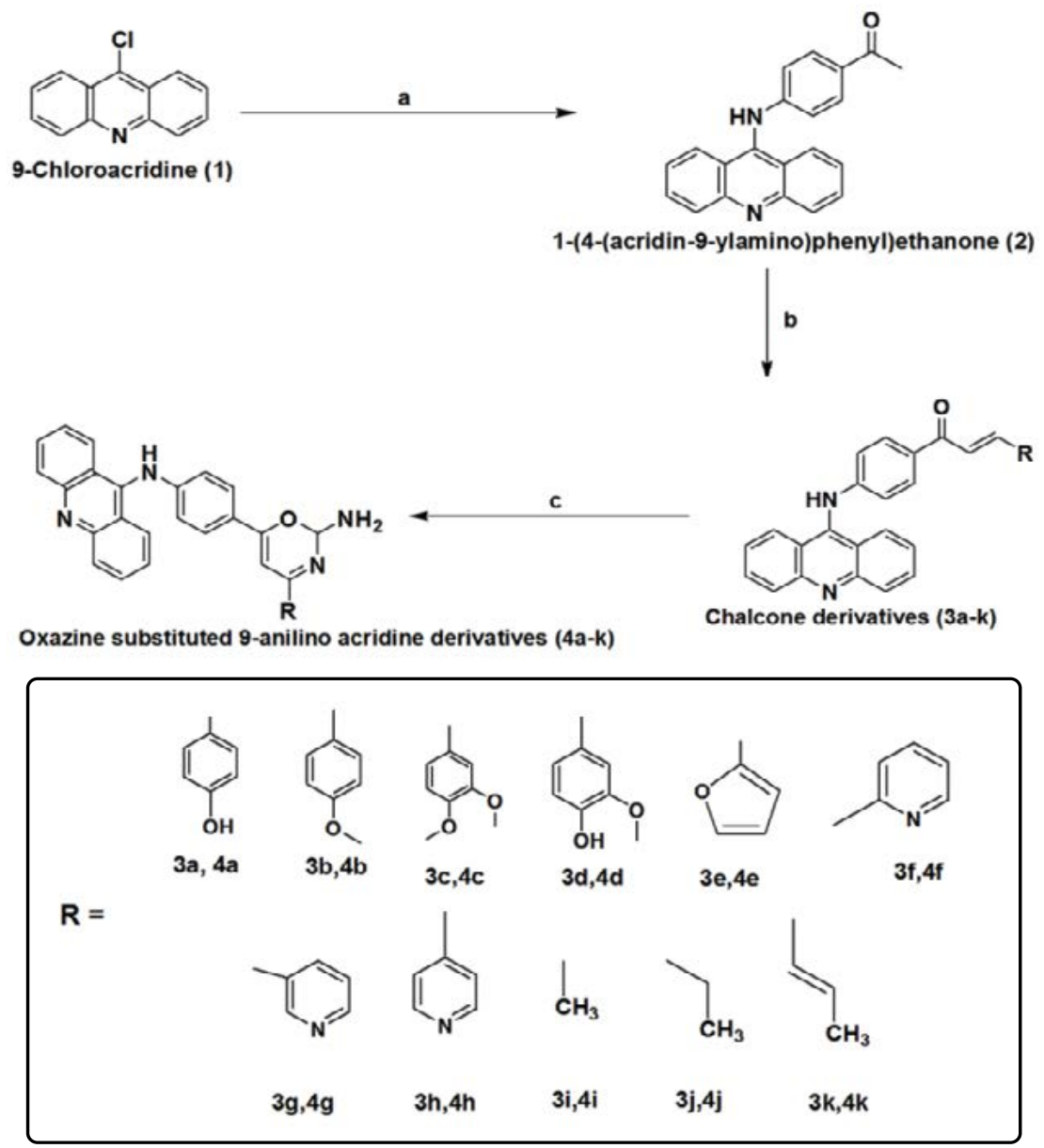

Fig. 1: Reaction sequences for oxazine substituted 9-anilinoacridines

a. 2-BuOH+4-aminoacetophenone, refluxed at $130-140^{\circ}$ for $3 \mathrm{~h}$ on an oil bath, b. EtOH+10\% $\mathrm{NaOH}+\mathrm{R}-\mathrm{CHO}$, stirred at room temperature for $8 \mathrm{~h}$ and c. cyclization with urea, stirred for 2-3 $\mathrm{h}$ 
The pharmacological properties of the compounds greatly depended on the number and the chemical nature of the substituents. The synthesized final

\begin{tabular}{|c|c|}
\hline Compound No. & $\mathrm{CTC}_{50}(\mu \mathrm{g} / \mathrm{ml})$ \\
\hline $4 a$ & 385 \\
\hline $4 \mathrm{~b}$ & 107 \\
\hline $4 c$ & 190 \\
\hline $4 d$ & 250 \\
\hline $4 e$ & 118 \\
\hline $4 f$ & 116 \\
\hline $4 g$ & 105 \\
\hline $4 \mathrm{~h}$ & 96.5 \\
\hline $4 \mathrm{i}$ & 140 \\
\hline $4 j$ & 115 \\
\hline $4 \mathrm{k}$ & 365 \\
\hline
\end{tabular}

DLA- Dalton's lymphoma ascites cells, $\mathrm{CTC}_{50}$ - concentration required to reduce viability by $50 \%$

\begin{tabular}{lcccc}
$\begin{array}{l}\text { TABLE 2: } \\
\text { AGAINST DLA CELLS }\end{array}$ & VIVO & ANTICANCER & ACTIVITY \\
\hline Group & $\begin{array}{c}\text { Dose } \\
(\mathbf{m g} / \mathbf{k g})\end{array}$ & Compound & $\begin{array}{c}\text { MST (In } \\
\text { Days) }\end{array}$ & \% ILS \\
\hline 2 (Control) & 10 & CMC (0.05\%) & $13 \pm 2.7$ & -- \\
3 (Standard) & 10 & 5 -Fluorouracil & $23.33 \pm 0.8$ & 79.46 \\
4 & 10 & $5 \mathrm{~b}$ & $17.33 \pm 1.6$ & 33.30 \\
5 & 20 & $5 \mathrm{~b}$ & $23.33 \pm 1.0$ & 79.46 \\
6 & 10 & $5 \mathrm{~h}$ & $19.33 \pm 1.0$ & 48.69 \\
7 & 20 & $5 \mathrm{~h}$ & $23.33 \pm 1.1$ & 79.46 \\
\hline
\end{tabular}

MST values are mean $\pm S D, n=6, M S T$ : mean survival time, $\%$ ILS: percent increase in life span
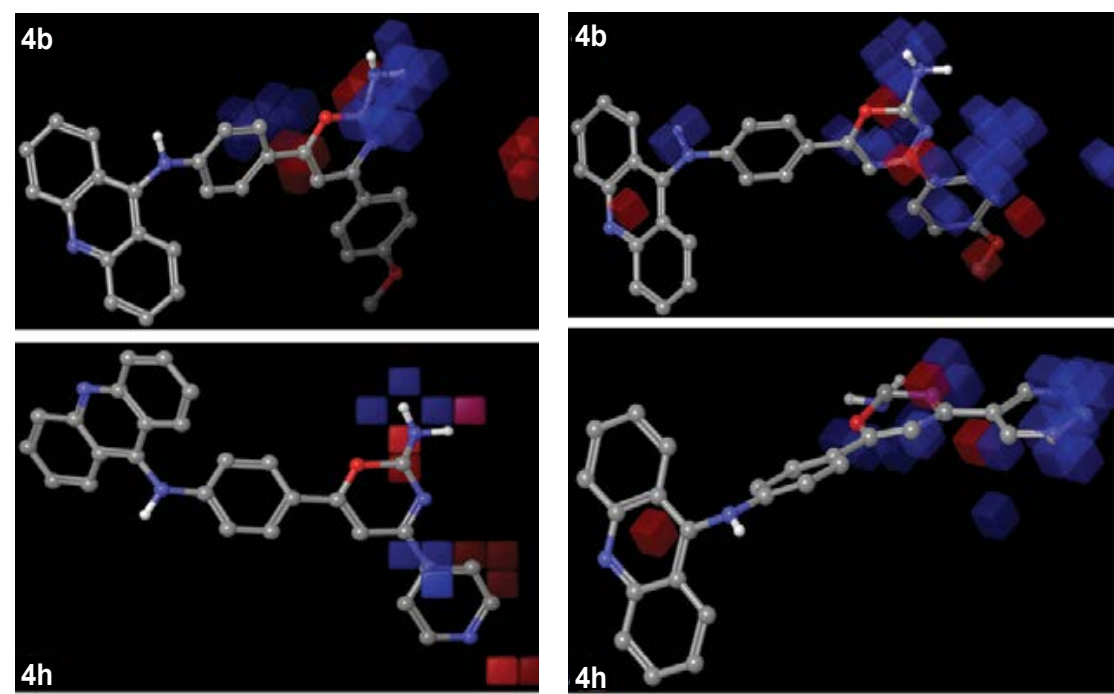

A

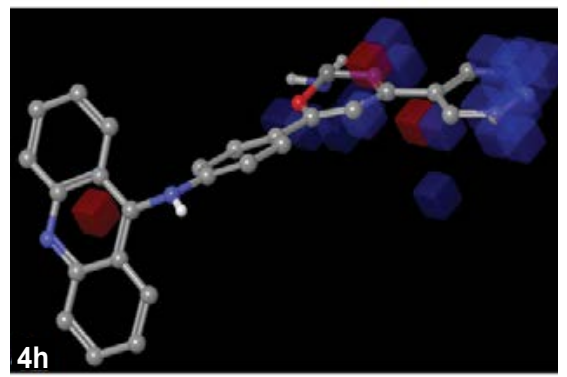

B compounds $4 \mathrm{a}-\mathrm{k}$ were subjected to short term study for in vitro antitumor activity against DLA cells. The compounds $4 \mathrm{~b}, 4 \mathrm{c}, 4 \mathrm{e}-4 \mathrm{j}$ exerted significant antitumour activity against DLA cells at the concentration of 96.5-190 $\mu \mathrm{g} / \mathrm{ml}(0.125-0.352 \mu \mathrm{M}$, Table 1).

The compounds $4 \mathrm{~b}$ and $4 \mathrm{~h}$ were screened further to evaluate in vivo antitumor activity against DLA cells. The in vivo study was carried out for $24 \mathrm{~d}$. Body weight gradually increased for many groups. The body weight analysis, MST and \% increase in life span at the dose of 10 and $20 \mathrm{mg} / \mathrm{kg}$ in Swiss albino mice inoculated with DLA cells $\left(1 \times 10^{6}\right)$ were calculated (Table 2$)$.

The 3D-QSAR model was generated by PHASE module of Schrodinger suite 2012. The predictive ability was analysed for the training set as well as the test set molecules. The features represented by the model with hydrogen bond donor, electron withdrawing group and hydrophobic/non-polar group (fig. 2). Blue colour region represented the favourable position for substitution and the red colour region represented the non-favourable position for substitution of groups.

In conclusion, acridine family includes a wide range of tricyclic molecules with various biological properties. Considered as potential antitumour agents since the 1980s, numerous acridine derivatives have been synthesised and successfully assessed for their antitumour activity. On this basis, authors recently demonstrated that diverse compounds of the oxazine substituted 9-anilinoacridine series exerted potent antitumour activities. It was revealed that these

Fig. 2: 3D QSAR model based on compounds $4 \mathrm{~b}$ and $4 \mathrm{~h}$

3D QSAR model for compounds $4 \mathrm{~b}$ and $4 \mathrm{~h}$ illustrates (A) hydrogen bond donor feature, (B) electron withdrawing group and (C) hydrophobic feature. The blue colour region represents favourable position for substitution and the red colour region represents non-favourable position for substitution 
agents exhibited significant cytotoxicity against DLA cell growth. Results observed in the present study clearly demonstrated that some derivatives of the oxazine substituted 9-anilinoacridine family could exert interesting antitumour activity. The compounds 4a-k showed significant antitumour activity and have the potential to be developed as useful drugs after further refinement. These derivatives certainly provide impetus to design future antitumour agents with greater therapeutic potential.

\section{Acknowledgements:}

We thank All India Council for Technical Education, New Delhi for the financial support under Research Promotion Scheme. We also thank our Vice Chancellor Dr. B. Suresh, JSS University, Mysore, our principal S. P. Dhanabal, Department of Pharmaceutical analysis, Department of Pharmaceutical Biotechnology, Department of Pharmacology, JSS College of pharmacy, Ooty for the technical support.

\section{Conflict of interest:}

There is no conflict of interest among authors.

\section{REFERENCES}

1. Fossé P, René B, Saucier JM, Hénichart JP, Waring MJ, Colson P, et al. Stimulation of Site-Specific Topoisomerase IIMediated DNA Cleavage by an N-Methylpyrrolecarboxamideanilinoacridine Conjugate: Relation to DNA Binding. Biochemistry 1994;33(33):9865-74.

2. Chilin A, Marzaro G, Marzano C, Dalla Via L, Ferlin MG, Pastorini G, et al. Synthesis and antitumor activity of novel amsacrine analogs: The critical role of the acridine moiety in determining their biological activity. Bioorg Med Chem 2009; 17:523-9.

3. Nadaraj V, Selvi ST, Mohan S. Microwave-induced synthesis and anti-microbial activities of 7,10,11,12-tetrahydrobenzo[c] acridin-8(9H)-one derivatives. Eur J Med Chem 2009;44:97680.

4. Kalirajan R, Muralidharan V, Jubie S, Gowramma B, Gomathy S, Sankar S, et al. Synthesis of some novel pyrazole substituted 9-anilino acridine derivatives and evaluation for their antioxidant and cytotoxic activities. J Heterocycl Chem 2012;49:748-54.

5. Santelli-Rouvier C, Barret JM, Farrell CM, Sharples D, Hill BT, Barbe J. Synthesis of 9-acridinyl sulfur derivatives: sulfides, sulfoxides and sulfones.Comparison of their activity on tumour cells. Eur J Med Chem 2004;39:1029-38.

6. Rastogi K, Chang JY, Pan WY, Chen CH, Chou TC, Chen LT, et al. Antitumor AHMA linked to DNA minor groove binding agents: synthesis and biological evaluation. J Med Chem 2002;45(20):4485-93.

7. Su TL, Chou TC, Kim JY, Huang JT, Ciszewska G, Ren WY, et al. 9-Substituted Acridine Derivatives with Long Half-
Life and Potent Antitumor Activity: Synthesis and StructureActivity Relationships. J Med Chem 1995;38:3226-35.

8. Bacherikov VA, Chang JY, Lin YW, Chen CH, Pan WY, Dong $\mathrm{H}$, et al. Synthesis and antitumor activity of 5-(9-acridinylamino)anisidine derivatives. Bioorg Med Chem 2005;13:6513-20.

9. Gamage SA, Tepsiri N, Wilairat P, Wojcik SJ, Figgitt DP, Ralph RK, et al. Synthesis and in vitro evaluation of 9-anilino3,6-diaminoacridines active against a multidrug-resistant strain of the malaria parasite Plasmodium falciparum. J Med Chem 1994;37(10):1486-94.

10. Chen YL, Lu CM, Chen IL, Tsao LT Wang JP. Synthesis and antiinflammatory evaluation of 9-anilinoacridine and 9-phenoxyacridine derivatives. J Med Chem 2002;45(21):4689-94.

11. Sondhi SM, Johar M, Nirupama S, Sukla R, Raghubir R Dastidar SG. Synthesis of sulpha drug acridine derivatives and their evaluation for anti-anflammatory, analgesic and anticancer activity. Indian J Chem 2002;41B:2659-66.

12. Gamage SA, Figgitt DP, Wojcik SJ, Ralph RK, Ransijn A, Mauel J, et al. Structure-activity relationships for the antileishmanialand antitrypanosomal activities of 1 '-substituted 9-anilinoacridines. J Med Chem 1997;40(16):2634-42.

13. Llama EF, Campo CD, Capo M, Anadon M. Synthesis and antinociceptive activity of 9-phenyl-oxy or 9-acyl-oxy derivatives of xanthene, thioxanthene and acridine. Eur J Med Chem 1989;24:391-6.

14. Recanatini M, Cavalli A, Belluti F, Piazzi L, Rampa A Bisi A, et al. SAR of 9-amino-1,2,3,4-tetrahydroacridine-based acetylcholinesterase inhibitors: synthesis, enzyme inhibitory activity, QSAR, and structure-based CoMFA of tacrine analogues. J Med Chem 2007;43(10):2007-18.

15. Goodell JR, Madhok AA, Hiasa H, Ferguson DM. Synthesis and evaluation of acridine- and acridone-based anti-herpes agents with topoisomerase activity. Bioorg Med Chem 2006;14:5467-80.

16. Harrison RJ, Cuesta J, Chessari G, Read MA, Basra SK, Reszka AP, et al. Trisubstituted acridine derivatives as potent and selective telomerase inhibitors. J Med Chem 2003;46(21):4463-76.

17. Mathew BP, Kumar A, Sharma S, Shukla PK, Nath M. An ecofriendly synthesis and antimicrobial activities of dihydro- $2 \mathrm{H}-$ benzo- and naphtho-1,3-oxazine derivatives. Eur J Med Chem 2010;45:1502-7.

18. Kalirajan R, Sivakumar SU, Jubie S, Gowramma B. Suresh B. Synthesis and biological evaluation of some heterocyclic derivatives of chalcones. Indian $\mathrm{J}$ Chem Tech Res 2009; 1(1):27-34.

19. Shen G, Chen D, Zhang Y, Sun M, Chen K, Jin C, et al. Synthesis of benzoxazine and 1,3-oxazine derivatives via ligand-free copper(I)-catalyzed one-pot cascade addition/ cyclization reaction. Tetrahedron 2012;68:166-72.

20. Basappa, Murugan S, Kavitha CV, Purushothaman A, Nevin $\mathrm{KG}$, Sugahara $\mathrm{K}$, et al. A small oxazine compound as an antitumor agent: A novel pyranoside mimetic that binds to VEGF, HB-EGF, and TNF- $\alpha$. Cancer Lett 2010;297:231-43.

21. Kalirajan R, Rafick MH, Sankar S, Jubie S. Docking studies, Synthesis, Characterization and Evaluation of their Antioxidant and cytotoxic activities of some Novel Isoxazole substituted 9-anilinoacridine derivatives. ScientificWorldJournal 2011;2012:165258.

22. Di Giorgio C, Shimi K, Boyer G, Delmas F, Galy JP. Synthesis 
and antileishmanial activity of 6-mono-substituted and 3,6-disubstituted acridines obtained by acylation of proflavine. Eur J Med Chem 2007;42:1277-84.

23. Kalirajan R, Rathore L, Jubie S, Gowramma B, Gomathy S, Sankar S, et al. Microwave assisted synthesis and evaluation of pyrazole derivatives of benzimidazoles. Indian $\mathrm{J}$ Chem 2011;50B:1794-9.

24. Kalirajan R, Kulshrestha V, Sankar S, Jubie S. Docking studies, synthesis, characterization of some novel oxazine substituted 9-anilinoacridine derivatives and evaluation for their anti-oxidant and anticancer activities as topo isomerase ii inhibitors. Eur J Med Chem 2012;56:217-24.

25. Kalirajan R, Palanivelu M, Rajamanickam V, Vinothapooshan G, Anandarajagopal K. Synthesis and biological evaluation of some chalcone derivatives. Int J Chem Sci 2007;5(1):73-80.

26. Vijayan P, Kumar S, Dhanaraj SA, Badami S, Suresh B. In vitro Cytotoxicity and Anti-tumor Properties of the Total Alkaloid Fraction of Unripe Fruits of Solanum pseudocapsicum. Pharm Biol 2002;40(6):456-60. 